\title{
Autosomal recessive polycystic kidney disease
}

INSERM

\section{Source}

INSERM. (1999). Orphanet: an online rare disease and orphan drug data base. Autosomal recessive polycystic kidney disease. ORPHA:731

A rare, genetic hepatorenal fibrocystic syndrome characterized by cystic dilatation and ectasia of renal collecting tubules, and a ductal plate malformation of the liver resulting in congenital hepatic fibrosis. Clinical presentation, whilst typically in utero or at birth, is variable and in the most severe cases includes Potter-sequence, oligohydramnios, pulmonary hypoplasia, and massively enlarged echogenic kidneys. 